\title{
Breaking away from trend-based analysis for regional modelling and planning
}

\author{
$\underline{\text { P. Perez }}^{\text {a }}$, R. Wickramasuriya ${ }^{\text {a }}$, H. Forehead ${ }^{\text {a }}$, F. Ramezani ${ }^{\text {a }}$ and J. Barthélemy ${ }^{\text {a }}$ \\ a SMART Infrastructure Facility, Faculty of Engineering and Information Sciences, University of \\ Wollongong, Australia \\ Email:pascal@uow.edu.au
}

\begin{abstract}
Traditional approaches to regional modelling and planning rely on trend-based analyses whereby demographic projections are used to initialise and bootstrap future demand for housing, transport and jobs. Besides, most of these approaches cannot adequately take into account the intricate interactions between land use, transport and regional economic drivers. The model developed for the Illawarra region (south of Sydney, Australia), called Vision Illawarra, draws upon and expands the Metronamica $C$ platform developed by RIKS (Netherlands) into a 4-component dynamic model. A regional economic model (CGE) drives the number of jobs available and informs a demographic model (Synthetic Population) on employment levels and migration needs. The land use model (cellular automaton) spatially allocates the residential, commercial and industrial areas based on demand set by the economic and population models and existing/future zoning plans. A 4-step transport model is informed by the land use model and provides a dynamic feedback on land accessibility. Vision Illawarra has been calibrated for the period $2006-2011$ and the simulation of scenarios uses the time window between 2011 and 2031. In this paper, we present the development and calibration of the model, as well as the exploration of three plausible scenarios with the model.
\end{abstract}

Keywords: $\quad$ Regional planning, integrated modelling, Vision Illawarra 


\section{INTRODUCTION}

Strategic regional planning concerns long term projects that aim to bring about sustainable development through insightful investment in and management of public and private resources (United Cities and Local Governments, 2010). This is often a challenging process as it requires careful balancing of competing priorities such as vibrant and connected communities, accessible and affordable transport, innovative and productive economy, and protected natural environments (Geerlings and Stead, 2003).

Modelling and simulation provides a mechanism to understand the complex inter-relationships and dynamic evolution of a regional system. However, many current regional planning models rely on feed-forward trend analysis. These trends are based on demographic or economic assumptions that lock-in regional growth into a unique pathway. A weaker initial assumption can result in a less plausible evolution in other aspects of the regional development. Regional planning models need to follow observed patterns to become useful. Further, most existing models used in regional planning are limited to isolated land use models or transport models. Model integration in a handful of existing studies has been limited to mostly two integrated components: land use and transport. Such models fail to account for the dynamic interactions between economic, demographic, land use and transport sub-systems of a regional system. This can result in having to work with a myriad of assumptions and unrealistic simulation outputs.

To this end, such models should incorporate land use, transport, economic and demographic factors that interact with each other and co-evolve in a simulation environment (van Delden et al, 2011). Vision Illawarra, the subject of this paper, is a fully integrated model that comprises of the foregoing key subsystems. Next we discuss the study area, setting up and calibration of the model, and the scenario exploration using Vision Illawarra.

\section{METHODOLOGY}

\subsection{Study area}

The Illawarra, a coastal region located south of Sydney in Australia, is our study area that consists of three Local Government Areas (LGAs): Wollongong, Shellharbour and Kiama (Figure 1). These three LGAs occupy the coastal plain limited on the east by a forested escarpment, hence geographically well-demarcated as a region. According to the 2016 census (Australian Bureau of Statistics, 2016), the population of the Illawarra region stands at 293,551 persons, $69 \%$ of which live in Wollongong LGA only.

\subsection{Setting up, calibration and validation}

We use an established Computable General Equilibrium Model (CGE) called Cadence Economics General Equilibrium Model (CEGEM - Office of the Chief Economist, 2016; SMART Infrastructure Facility, 2017) as the economic model of Vision Illawarra. CEGEM is a multi-commodity, multi-region, dynamic model of the world economy. Like all economic models, CEGEM is a based on a range of assumptions, parameters and data that constitute an approximation to the working structure of an economy. Its construction has drawn on the key features

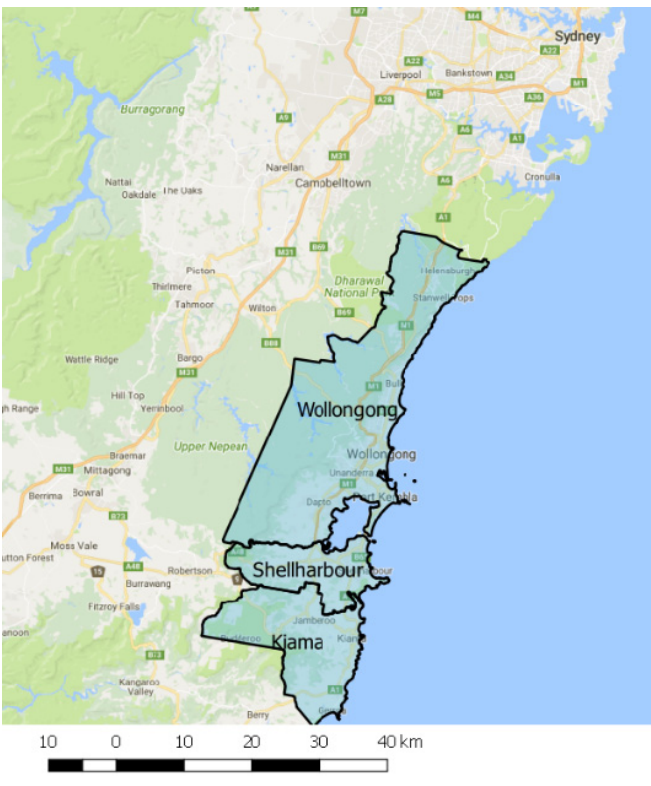

Figure 1. Study area: The Illawarra Region, New South Wales, Australia of other economic models such as the global economic framework underpinning models such as GTAP and GTEM, with state and regional modelling frameworks such as Monash-MMRF and TERM. CEGEM model calculates the yearly growth or decline of jobs for 19 economic sectors including agriculture, mining, industrial, health and education. These job numbers are then fed to the synthetic population model, which in turn estimates the migration into the region. Further, job numbers for four aggregated economic sectors (commercial and service, industrial, agriculture and mining) are fed into the land use model which then spatially allocates those jobs.

Synthetic population model follows a sample-free approach to synthesise the population of the Illawarra region. Different to existing sample-free algorithms, the population synthesizer applies the heuristics to part 
of the allocation of synthetic individuals into synthetic households. As a result the iterative process of allocating individuals into households, which normally is the most computationally demanding and time consuming process, is required only for a subset of synthetic individuals. The population synthesiser is therefore computationally efficient allowing us to build a large synthetic population. This capability ensures that the geographical heterogeneity of the resulting synthetic population is best preserved. Further details of the configuration, calibration and validation of the synthetic population is discussed by Huynh et al. (2016). Synthetic population model simulates population dynamics by implicitly modelling births, deaths, marriages and migration into and out of the study area. Availability of jobs in the region as estimated by the CEGEM model acts as a major catalyst for migration. Total population of the Illawarra region generated by the synthetic population is fed into the land use model, which in turn spatially allocates this demand into residential land use types.

We use Metronamica framework (Van Delden and Engelen, 2006) to set up land use and transport models. The constrained Cellular Automata (CA) based land use model in Metronamica has been used in many applications around the world, both in the original form and its variants (Wickramasuriya et al., 2009; Stanilov and Batty, 2011; van Vliet et al., 2012). The main variable of the land use model is a land use map in raster format which is iteratively updated in yearly time steps over the course of the simulation. Land use transitions are driven by endogenously calculated transition potential for which neighbourhood effect, physical suitability, accessibility (mainly to transport) and zoning contribute as key factors. A comprehensive description of the land use model is provided by RIKS BV (2005) and Wickramasuriya et al. (2009). Transport model is based on a classical four step approach (McNally, 2000), but has been made dynamic (RIKS BV, 2005). The land use model provides input to determine origins and destinations of trips, while transport model influences land use change by means of accessibility. This two-way communication provides the dynamic basis to the transport model.

The land use classification used in this study includes 23 classes out of which 6 are dynamic. The dynamic classes include medium density residential, low density residential, commercial and services, industrial, agriculture and mining. Spatial resolution used in the land use model is $100 \mathrm{~m}$. Travel zones as identified by the Bureau of Transport Statistics (BTS) NSW are used as transport zones. We model 5 time periods of a

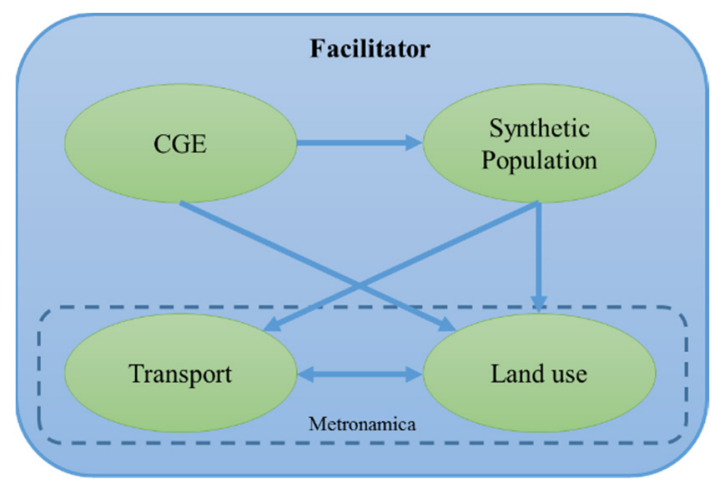

Figure 2. Vision Illawarra integrated model typical weekday (midnight, morning peak, noon, afternoon peak and evening), two modes (car and public transport) and 4 purposes (work, shopping, social and home). A multi-dimensional Origin Destination (OD) matrix for all travel zone pairs in the region is prepared using the Household Travel Survey data gathered by BTS.

All foregoing models are brought together by a module called 'facilitator', which is developed using python programming language. Facilitator is responsible for the orderly execution of individual models and passing input/output data between modules. It also collects final outputs from all models, organises and saves this data for later use.

Vision Illawarra is calibrated for the period 2006 to 2011. Calibration and validation of Vision Illawarra is discussed at length by Wickramasuriya et al. (2014).

\subsection{Scenario exploration}

We used Vision Illawarra to explore three plausible scenarios: baseline, alternative zoning and road improvement.

- Baseline scenario simulates the economic, demographic, land use and transport dynamics of the Illawarra region between 2011 and 2031, assuming current trends will continue undisturbed.

- Alternative zoning scenario explores the impact of allowing residential densification in the 'Northern Corridor', which corresponds to a strip of land along Princes highway between Lake Illawarra in the south and Helenburg in the north. This strip of land currently dominated by low density residential land use can be converted into medium density residential land use under this policy scenario.

- Road improvement scenario concerns a proposed project called 'Albion Park bypass' that is aimed at reducing peak congestion on Princes highway at Albion Park. Albion Park pinch point is largely responsible for the peak travel time of about 1 hour between Kiama and Wollongong. Peak travel time 


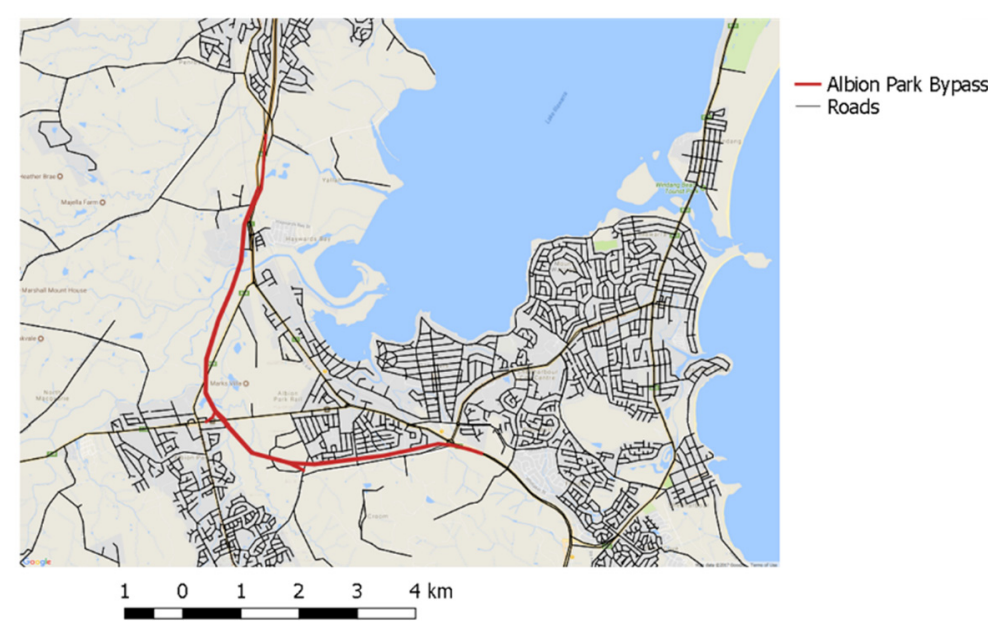

between these two cities is about 25 minutes greater than off-peak travel time. In this scenario, we explore the effectivenss of the proposed Albion Park bypass in reducing congestion on Princes highway. Figure 3 illustrates the new road alignment used in this scenario. It is worth noting that the alignment we use in this exercise may differ from the actual alignment.

Figure 3. Albion Park bypass alignment

\section{RESULTS}

\subsection{Baseline scenario}

Although the total job number in the Illawarra region grows linearly, most of this growth comes from the commercial and service sector at large. Industrial and mining sectors continue to lose jobs as shown in Figure 4. By 2031, industrial sector would lose about $30 \%$ of its current jobs under the present trend. In short, Illawarra region is transitioning from a manufacturing into a service economy.

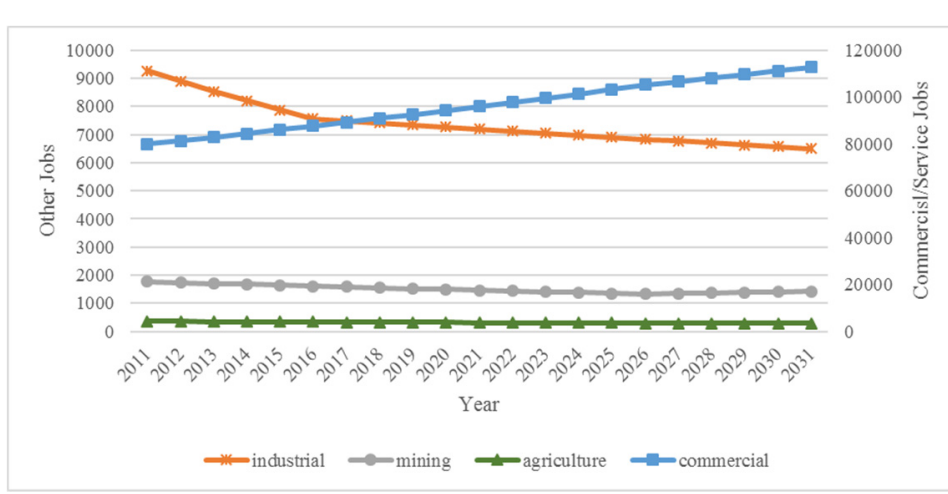

Figure 4. Change in jobs between 2011 and 2031 as forecasted by the CGE model (4 categories)
As simulated by the synthetic population, the population of Illawarra rises from 256,164 in 2011 to 381,236 in 2031 in a linear fashion. Additionally, we compared the simulated population and the census figures for 2016 as a point of validation. These figures are 285,941 and 293,551 respectively for synthetic population and the 2016 census. Synthetic population slightly underestimates the total population, but the deviation is an acceptable $2.6 \%$.

Land use model respects the decreasing industrial jobs as evident by significant extent of industrial land converted into vacant and other land uses. It is worth noting that the abandoned industrial sites do not get immediately converted into residential or commercial land uses despite their prime location. Land use model has been configured to account for the time it takes to decontaminate industrial sites before reuse. New residential and commercial land uses appear in the West Dapto Calderwood areas that are zoned for green field development. Some low density residential areas in the Wollongong CBD give way to medium density residential land use. Figure 5 illustrates the land use change between 2011 and 2031 for four key land use classes. 


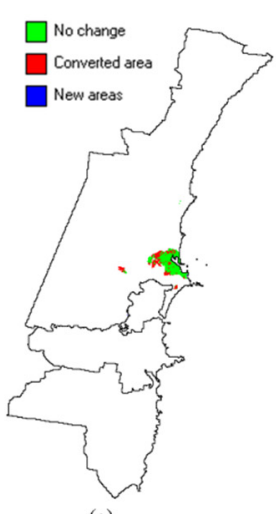

(a)

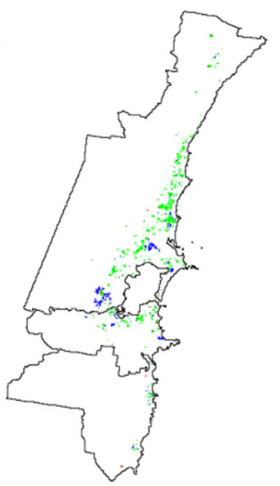

(b)

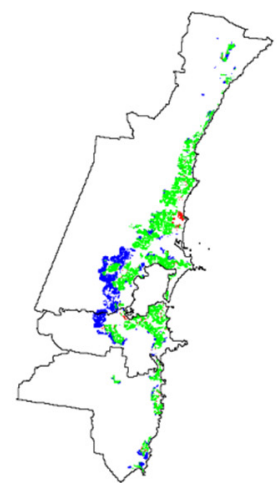

(c)

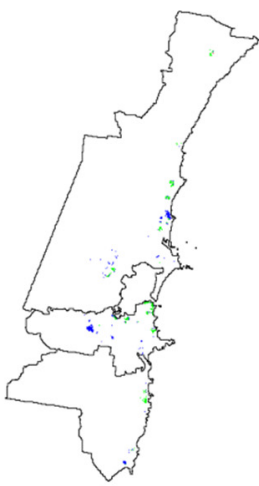

(d)

Figure 5. Land use change between 2011 and 2031, (a) industrial, (b) commercial and services, (c) low density residential, and (d) medium density residential

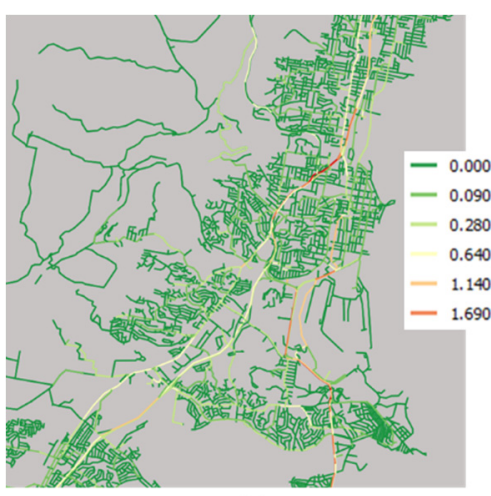

(a)

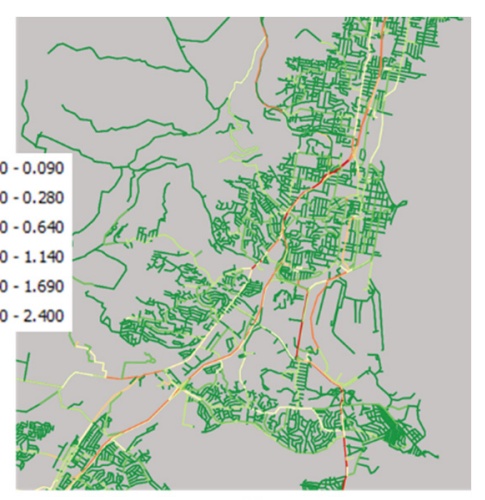

(b)

Figure 6. Change in maximum road congestion, (a) 2011, (b)

With no major improvements to the road network, average travel time during peak period increases significantly between 2011 and 2031 . For example, the travel time between Kiama and Wollongong during the morning peak goes up by about 30 minutes from the current average time of 48 minutes. Network congestion increases substantially, particularly on major highways (Figure 6).

\subsection{Densification scenario}

Under the densification scenario, low density residential land along the northern corridor is giving way to medium density residential use (Figure 7). More importantly, densification policy effectively directs the scattered new medium density development that is seen in the baseline into the densification corridor (Figure 8).

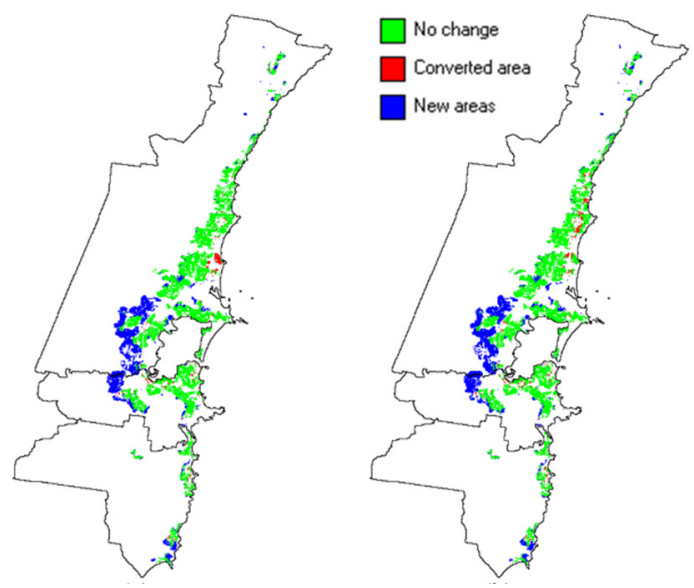

Figure 7. Change in low density residential area between 2011 and 2031, (a) baseline, (b) densification 


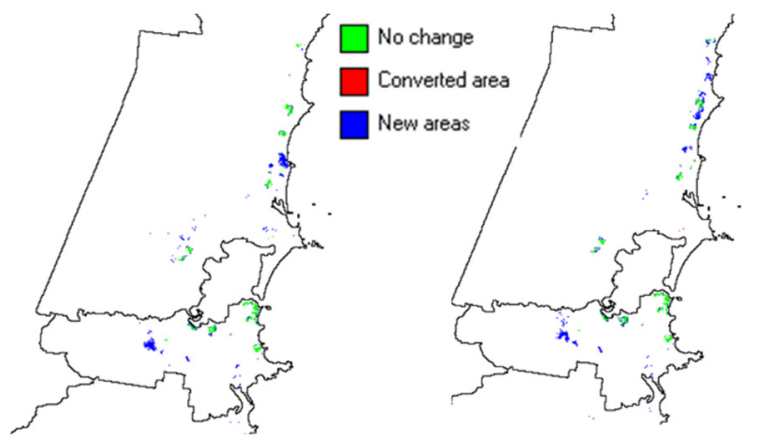

(a)

(b)

\subsection{Road infrastructure scenario}

Peak morning travel from Kiama to Wollongong is usually the worst time for a motorist. Travel time of this trip is a good indicator to assess the effectiveness of the Albion Park bypass in reducing congestion and improving travel time. Figure 9 compares the travel time for this particular trip between the baseline and road improvement (bypass) scenario for the period $2011-2031$.

Figure 8. Change in medium density residential area between 2011 and 2031, (a) baseline, (b) densification

As expected, both scenarios show an increasing travel time for the concerned trip due to the growth of population and mobility demand. As soon as the Albion Park bypass is open in year 2023, it immediately brings the travel time down by about 10 minutes. More importantly, the time gap between the baseline and bypass scenarios widens as the simulation progresses, leaving this difference at 20 minutes by 2031 .

Vision Illawarra is an ongoing research project. Our current efforts focus mainly on capturing and enhancing the feedback loops between modules. This work and further scenario exploration are the subjects of a future paper.

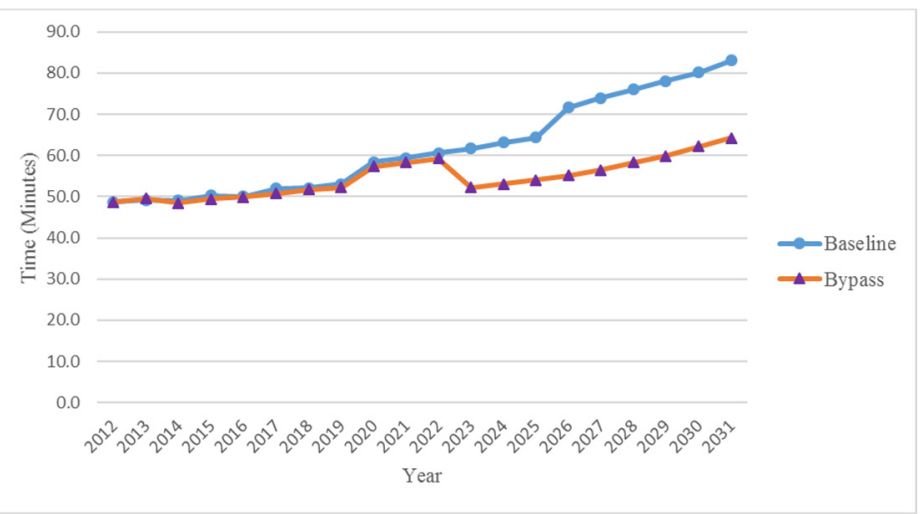

Figure 9. Comparison of travel time change between baseline and bypass scenarios for an average trip from Kiama to Wollongong during morning peak

\section{CONCLUSION}

We developed an integrated planning model called Vision Illawarra that comprises of four sub-models: CGEbased economic model, synthetic population, CA-base land use model and 4-step transport model. This model was configured, calibrated and validated for the Illawarra region, an area of south of Sydney, Australia. Vision Illawarra deviates substantially from traditional panning models as it models aforementioned key sub-systems of a complex urban system, including the dynamic feedbacks between the sub-systems. Hence, Vision Illawarra is capable of testing the impact of economic, demographic, zoning and transport policies. This capability provides two main benefits to planners and policy makers, (a) the ability to test the flow on effect of a policy on other inter-connected systems, and (b) a single tool to run diverse set of policy scenarios.

In this study, we used Vision Illawarra to explore three plausible scenarios. Baseline scenario assumed the current economic and demographic trends to continue undisturbed, no upgrades to road infrastructure, and no changes to the zoning policies. Densification scenario considered an alternative zoning option where high value land along a major road corridor is opened up for medium density residential use. Third scenario explored the potential impact of a proposed bypass road on road network congestion and peak travel time. Densification policy tend to be successful in directing otherwise scattered medium density residential development into a defined corridor with greater access. Simulation results suggest the proposed bypass may provide a substantial travel time saving during peak hours. 
Perez et al., Breaking away from trend-based analysis for regional modelling and planning

Ongoing research focuses on improving dynamic feedback loops between models, and exploring various policy scenarios chosen by the stakeholder groups.

\section{ACKNOWLEDGMENTS}

Authors wish to acknowledge the data providers and stakeholders of the Vision Illawarra project, particularly Wollongong, Shelharbour and Kiama city councils, Spatial Services division of the NSW Department of Finance, Service and Innovation, Sydney Water, Endeavour Engergy and REMONDIS.

\section{REFERENCES}

Australian Bureau of Statistics. (2016). The Census of Population and Housing.

Geerlings, H., and Stead, D. (2003). The integration of land use planning, transport and environment in European policy and research. Transport Policy, 10(3), 187-196.

Huynh N., Perez P., Berryman M., Barthélemy J. (2015). Simulating Transport and Land Use Interdependencies for Strategic Urban Planning - An Agent-Based Modelling Approach Modelling Approach. Systems, 3 (4): 177-210.

Office of the Chief Economist. (2016). Australian Industry Report 2016. Department of Industry, Innovation and Science.

RIKS BV. (2005). METRONAMICA Documentation, RIKS BV, Maastricht, NL, 266p.

SMART Infrastructure Facility. (2017). Upgrading rail connectivity between Illawarra and Sydney. 68p.

Stanilov, K., and Batty, M. (2011). Exploring the Historical Determinants of Urban Growth Patterns through Cellular Automata. Transactions in GIS, 15(3), 253-271.

United Cities and Local Governments. (2010). Policy Paper on Strategic Urban Development. 6p.

van Vliet, J., Hurkens, J., White, R., and van Delden, H. (2012). An activity-based cellular automaton model to simulate land-use dynamics. Environment and Planning B-Planning \& Design, 39(2), 198-212.

van Delden, H., Seppelt, R., White, R., and Jakeman, A.J. (2011). A methodology for the design and development of integrated models for policy support. Environmental Modelling \& Software, 26(3), 266279.

van Delden, H., and Engelen, G. (2006). Combining participatory approaches and modelling: Lessons from two practical cases of policy support. $3^{\text {rd }}$ International Congress on Environmental Modelling and Software, Burlington, Vermont, USA, 9 - 13 July, 2006.

Wickramasuriya, R. C., Bregt, A. K., van Delden, H., and Hagen-Zanker, A. (2009). The dynamics of shifting cultivation captured in an extended Constrained Cellular Automata land use model. Ecological Modelling, 220(18), 2302-2309.

Wickramasuriya, R., Perez, P., Huynh, N., and van Delden, H. (2014). Facilitating Strategic Planning in Coastal Regions Through Integrated Modelling and Simulation. 23 ${ }^{\text {rd }}$ NSW Coastal Conference, Shoalhaven, NSW, 11 - 14 November 2014. 